\title{
Novel Peptide Nucleic Acid Melting Array for the Detection and Genotyping of Toxoplasma gondii
}

\author{
Soo Hwan Suh ${ }^{1}$, Han Seong Yun ${ }^{1}$, Sang-Eun Lee ${ }^{2}$, and Hyo-Sun Kwak ${ }^{1 *}$ \\ ${ }^{1}$ Food Microbiology Division, National Institute of Food and Drug Safety Evaluation, Ministry of Food and Drug Safety, Cheongju 28159, \\ Republic of Korea \\ ${ }^{2}$ Center for Laboratory Control of Infectious Diseases, Centers for Diseases Control \& Prevention, Cheongju 28159, Republic of Korea
}

Received: April 15, 2019 / Revised: May 24, 2019 / Accepted: May 24, 2019

\begin{abstract}
Despite differences in virulence between strains of Toxoplasma gondii, rapid and accurate genotyping methods are lacking. In this study, a method was developed to detect and genotype $T$. gondii in food and environmental samples using PCR and a novel peptide nucleic acid (PNA) melting array. An alignment of genome sequences for $T$. gondii type I, II, and III obtained from NCBI was generated, and a single nucleotide polymorphism analysis was performed to identify targets for PCR amplification and a PNA melting array. Prior to the PNA melting array, conventional PCR was used to amplify GRA6 of T. gondii. After amplification, the PNA melting array was performed using two different PNA hybridization probes with fluorescent labels (FAM and HEX) and quenchers. Melting curves for each probe were used to determine genotypes and identify mutations. A 214-bp region of the GRA6 gene of T. gondii was successfully amplified by PCR. For all $T$. gondii strains (type I, II, and III) used to evaluate specificity, the correct genotypes were determined by the PNA melting array. Non-T. gondii strains, including 14 foodborne pathogens and 3 protozoan parasites, such as Giardia lamblia, Cryptosporidium parvum, and Entamoeba histolytica, showed no signal, suggesting that the assay has a high specificity. Although this is only a proof-of-concept study, the assay is promising for the fast and reliable genotyping of $T$. gondii from food and environmental samples.
\end{abstract}

Keywords: Toxoplasma gondii, genotype, peptide nucleic acid, melting array, GRA6, PCR

\section{Introduction}

Peptide nucleic acid (PNA) probes, developed in the early 1990s, are DNA mimics, in which the negatively charged sugar-phosphate backbone is replaced by an achiral, neutral polyamide backbone formed by repetitive $N$-(2-aminoethyl) glycine units [1]. PNA can hybridize to complementary nucleic acid targets according to the Watson-Crick base-pairing rules [2]. Their unique chemical characteristics have been studied extensively owing to their research and diagnostic applications, e.g.,

\section{*Corresponding author}

Tel: +82-43-719-4301, Fax: +82-43-719-4300

E-mail: kwakhyos@korea.kr

() 2019, The Korean Society for Microbiology and Biotechnology in genomics, human pathology, virology, mycology, and bacteriology [3]. PNA molecules have unique hybridization characteristics; they exhibit rapid and strong binding to complementary targets and a lack of electrostatic repulsion [4].

Toxoplasma gondii, an opportunistic intracellular parasite, has the ability to infect any eukaryotic cell. It can infect all warm-blooded mammals, including humans [5]. Felids are the definitive hosts of $T$. gondii. Environmental contamination is commonly caused by the secretion of oocysts, which become infective upon sporulation, by felids. Intermediate hosts can be infected by $T$. gondii and produce tissue cysts in a number of organs, such as the skeletal muscle and central nervous system. When ingested in raw or uncooked food, these tissue cysts are 
also infectious for other intermediate host species, including humans. The rapid detection of $T$. gondii in animals and meat products typically depends on immunogenic assays, such as ELISA; however, a number of PCR amplification assays have recently been developed. Despite the development of effective PCR and real-time PCR assays for the identification of $T$. gondii in animals and meat products, methods for genotyping $T$. gondii strains (type I, II, and III) are limited to serological tests. Thus, a fast and reliable sequence-based assay is necessary for differentiating the acute strain (type I) from chronic virulent type II and III strains. In this study, we developed a PCR method for $T$. gondii detection in retail meat samples and performed a genotyping analysis using a novel PNA melting array.

\section{Materials and Methods}

\section{Strains}

T. gondii stains used in this study were RH (ATCC 50147, type I), ME49 (ATCC 50611, type II), PTG (ATCC 50941, type II), and STRL (ATCC 50955, type III). One Korean isolate (KI-1, type I) [6] was kindly provided by the KCDC (Korea Center for Disease Control and Prevention). DNA was extracted using an automated nucleic acid extraction system, NucliSENS ${ }^{\circledR}$ easyMAG ${ }^{\circledR}$ (bioMérieux, USA) according to the manufacturer's instructions.

Table 1. GRA6 gene region of Toxoplasma gondii used to make consensus sequences (NCBI).

\begin{tabular}{ccc}
\hline Accession No. & Strain (GRA6) & Type \\
\hline AF239283 & & \\
JX044182 & & \\
HM776942 & RH & Type I \\
AJ635332 & & \\
JN649063 & & \\
JX044183 & GT1 & Type II \\
AF239285 & & \\
JX044213 & ME49 & \\
XM_002371898 & & Type III \\
\hline JX044207 & CTG & \\
JX044209 & VEG & \\
AF239286 & NED & \\
\hline
\end{tabular}

\section{Primers and probes for PCR and the PNA melting array}

A 214-bp region of GRA 6 was selected for PCR amplification and a subsequent PNA melting array. To design primers and PNA probes, GRA6 genome of $T$. gondii type I, II, and III was obtained from the National Center for Biotechnology Information (Table 1). Consensus sequences were built using CLC Genomics Workbench (ver. 9.0.1.) and aligned using the online tool Clustal Omega (http://www.clustal.org).

\section{GRA6 gene amplification and the PNA melting array}

The GRA6 gene amplification and subsequent PNA melting array were performed with the protocol proposed by Kim et al. (2017) with minor modifications [7]. $T$. gondii DNA was amplified using forward and reverse primers (Forward 5'-CTT GGG AGT GTC GGC GAA ATG GCA C; Reverse 5'-CTG CTT CTT GCT GTC CAC CGC TCG) with a three-step thermocycling protocol as follows: initial denaturation at $95{ }^{\circ} \mathrm{C}$ for $10 \mathrm{~min}$, followed by 45 cycles of $95^{\circ} \mathrm{C}$ for $30 \mathrm{~s}, 58^{\circ} \mathrm{C}$ for $45 \mathrm{~s}$, and $72^{\circ} \mathrm{C}$ for $40 \mathrm{~s}$. The $20-\mu \mathrm{l}$ PCR volume contained freeze dried premix (AccuPower ${ }^{\circledR}$ PCR PreMix, Bioneer, Korea), 500 nM forward primer, $500 \mathrm{nM}$ reverse primer, and $2.5 \mu \mathrm{l}$ of $T$. gondii DNA. PCR was performed using the C1000 Touch $^{\text {TM }}$ Thermal Cycler (Bio-Rad, USA). After amplification, the PNA melting array was utilized for genotyping using the CFX96 ${ }^{\mathrm{TM}}$ real-time system (Bio-Rad) with two PNA hybridization probes (GRA6-1, 5'-FAM-GAG TCT ACC TAC GG-DABCYL-3'; GRA6-2, 5'-HEX-TGG GTA CTT CTG ATG T-DABCYL-3'). For the PNA melting array, initial activation to generate anti-sense DNA was performed at $95^{\circ} \mathrm{C}$ for $10 \mathrm{~min}$, followed by 15 cycles of $95^{\circ} \mathrm{C}$ for $30 \mathrm{~s}, 58^{\circ} \mathrm{C}$ for $45 \mathrm{~s}$, and $72^{\circ} \mathrm{C}$ for $40 \mathrm{~s}$. Subsequent PNA hybridization $\left(95^{\circ} \mathrm{C}\right.$ for $3 \mathrm{~min}, 75^{\circ} \mathrm{C}$ for $1 \mathrm{~min}$, and $55^{\circ} \mathrm{C}$ for $1 \mathrm{~min}$ ) and a melting temperature analysis $\left(40-85^{\circ} \mathrm{C}\right.$, step and hold, increase $1^{\circ} \mathrm{C}$ every $\left.10 \mathrm{~s}\right)$ were

Table 2. Coded table for calling Toxoplasma gondii genotypes.

\begin{tabular}{|c|c|c|}
\hline \multirow{2}{*}{ Types } & \multicolumn{2}{|c|}{ Call } \\
\hline & FAM & HEX \\
\hline Type I) & 1 & 1 \\
\hline Type $\|^{2)}$ & 0 & 1 \\
\hline Type III3) & 1 & 0 \\
\hline $\begin{array}{l}\text { and HEX } \\
\text { and HEX } \\
\text { and HEX }\end{array}$ & $\begin{array}{l}\text { (e.g. } \\
\text { (e.g. } \\
\text { (e.g. }\end{array}$ & \\
\hline
\end{tabular}




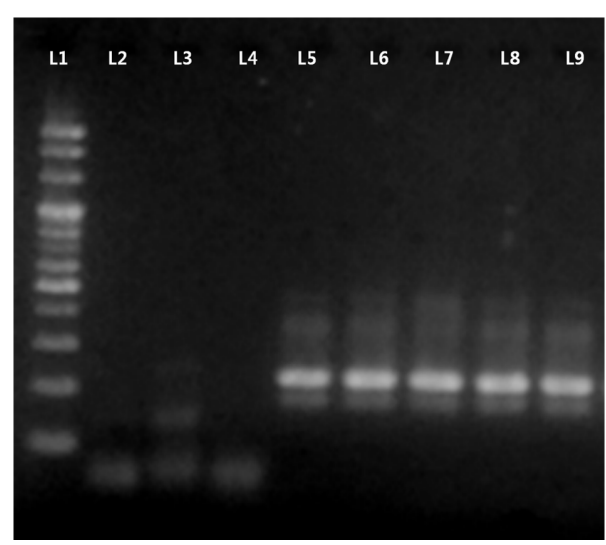

Fig. 1. PCR amplification of T. gondii strains and non-T. gondii strains. $※$ L1: 100K Ladder, L2: Cryptosporidium parvum, L3: Giardia lamblia, L4: Entamoeba histolytica, L5: T. gondii Type 1 (RH), L6: Type 1 (KI-1), L7: Type 2 (PTG), L8: Type 2 (ME49), L9: Type 3 (STRL)

performed in the same reaction tube. Reaction volumes of $20 \mu \mathrm{l}$ contained $10 \mu \mathrm{l}$ of melting array buffer (Seasunbio, Korea), 500 nM PNA probes (GRA6-1 and GRA6-2), and $500 \mathrm{nM}$ reverse primer (5'-CTG CTT CTT GCT GTC CAC CGC TCG) and $5 \mu \mathrm{l}$ of pre-amplified $T$. gondii DNA. After the PNA melting array, the genotypes of $T$. gondii strains were determined according to the codes shown in Table 2.
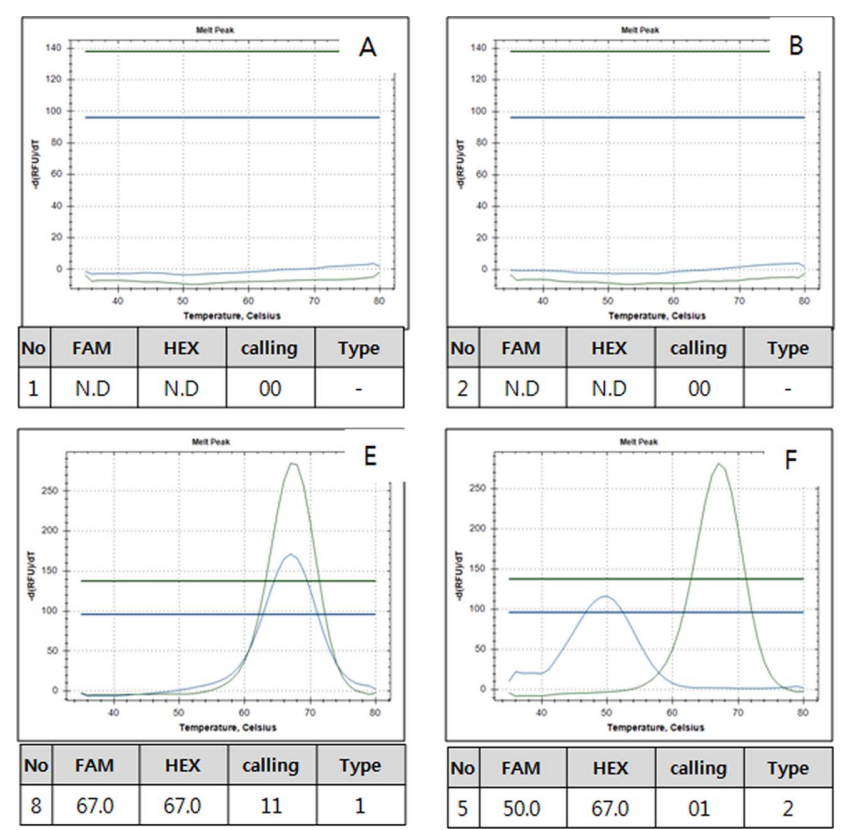

\section{Results}

Prior to the PNA melting array, GRA6 of $T$. gondii was amplified by PCR. In this analysis, all types of $T$. gondii strains showed clear bands at the expected size, i.e., 214 bp. Bands were not observed for non-T. gondii strains including Cryptosporidium parvum, Giardia lamblia and Entamoeba histolytica (Fig. 1).

To evaluate sensitivity, DNAs extracted from type 1 (RH; KI-1), type 2 (ME49; PTG), and type 3 (STRL) $T$. gondii strains were diluted 10-fold (5 ng to $0.00005 \mathrm{ng}$ ) and subjected to PCR amplification. The lower limit of detection for the $T$. gondii strains $\mathrm{RH}$ and KI-1 (type 1), ME49 (type 2), and STRL (type 3), was $5 \times 10^{4} T$. gondii DNA copies, while that for PTG (type 2) was $5 \times 10^{3} T$. gondii DNA copies (Supplemental Fig. 1).

Downstream genotyping was achieved using the PNA melting array based on a previously established SNP analysis [8]. Differences in $\Delta \mathrm{T}_{\mathrm{m}}$ values between perfect and mismatched probes were used to determine genotypes according to a calling table, as described in Table 2. As described in Table 3, " 1 " was assigned when the $\mathrm{T}_{\mathrm{m}}$ value was $65-69^{\circ} \mathrm{C}$ and "0" was assigned when the $\mathrm{T}_{\mathrm{m}}$ was less than $64^{\circ} \mathrm{C}$. Based on $\Delta \mathrm{T}_{\mathrm{m}}$, genotypes can be easily determined. For example, RH and KI-1 (type 1)
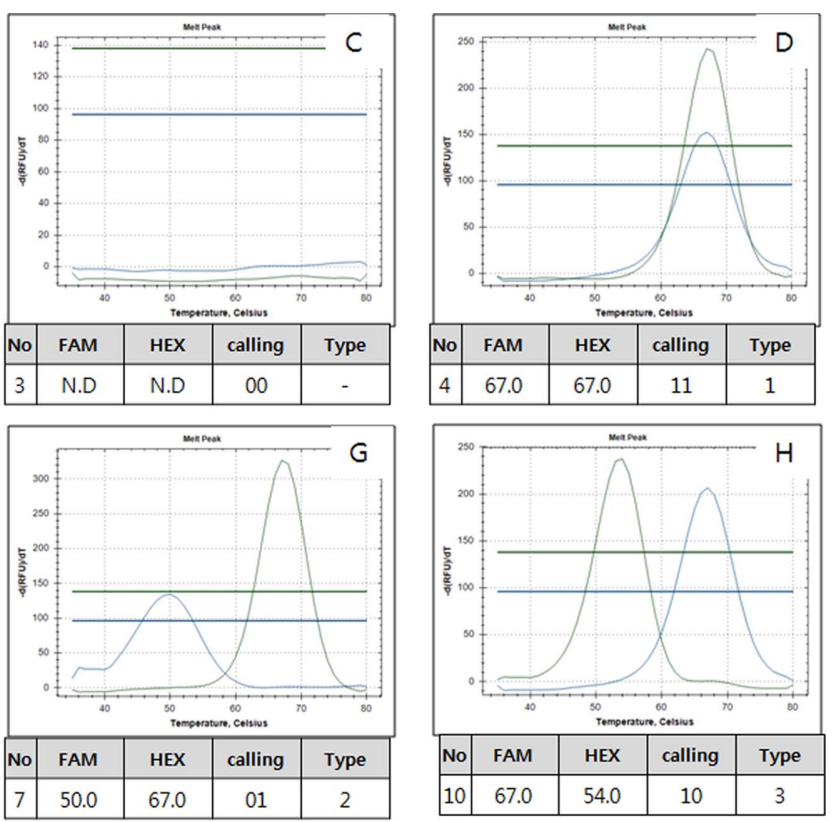

Fig. 2. Melting temperature analysis for the peptide nucleic acid melting array. ※ A: Cryptosporidium parvum, B: Giardia lamblia, C: Entamoeba histolytica, D: T. gondii RH, E: T. gondii KI-1, F: T. gondii PTG, G: T. gondii ME49, H: T. gondii STRL 
showed perfect matches for both FAM and HEX probes $($ call $=1,1)$, while ME49 (type 2) and STRL (type 3) showed only one perfect match for the HEX probe (call = $0,1)$ and FAM probe (call $=1,0)$ respectively.

As shown in Fig. 2, no melting peak was observed for non-T. gondii strains. Fig. $2 \mathrm{~A}, \mathrm{~B}$, and $\mathrm{C}$ represent $\mathrm{T}_{\mathrm{m}}$ values for Cryptosporidium parvum, Giardia lamblia, and Entamoeba histolytica, respectively. Type 1, 2, and 3 $T$. gondii strains showed calls of FAM $=1$ and $\mathrm{HEX}=1$; $\mathrm{FAM}=0$ and $\operatorname{HEX}=1 ;$ and $\mathrm{FAM}=1$ and $\operatorname{HEX}=0$, respectively $(\mathrm{E}-\mathrm{H})$. All $T$. gondii strains exhibited the correct genotypes, as determined by an analysis of melting temperatures.

\section{Discussion}

Although toxoplasmosis is not a major issue in Korea, it is a major threat to human health in Europe and North America. Dubey et al. [9] reported that human infections with $T$. gondii are common worldwide, with infection rates of $16 \%$ to $40 \%$ in the US and UK, and $50 \%$ to $80 \%$ in Central America, South America, and Europe. Song et al. [10] reported an antibody retention rate of $0.88 \%$ in an analysis of the serum and amniotic fluid of pregnant women in Korea. Kim et al. [11] suggested that the low infection rate in Korea can be explained by the relatively small number of households with cats and the low consumption of raw or uncooked pork. Cheong et al. [12] found that people who are potentially exposed to toxoplasmosis, such as workers in the livestock and meat processing industry, have a higher antibody retention rate.

Jones and Dubey [13] summarized antibody retention rates for toxoplasmosis in animals in the US from 1960 to 2012 , and observed rates of $0.3-92.7 \%$ in pigs, $4-$ $77.9 \%$ in sheep, $25.8 \%$ in goats, $0 \%$ in chickens, $0 \%$ in cattle, $17-28.7 \%$ in deer, and $35.7-70 \%$ in black bear. In addition, Racka et al. [14] reported a sero-prevalence of $30-40 \%$ for wild boar in the Czech Republic. In Korea, Kim et al. [11] reported that the retention rates of $T$. gondii antibodies for domestic cattle and pig were 4.6$20.7 \%$ and $16.8-22.9 \%$ respectively.

Investigations of toxoplasmosis in animals are mainly based on immunological assays (e.g., ELISA). Antibody positivity is measured by detecting $T$. gondii antibodies in the blood. Positivity from past exposure to $T$. gondii is thought to be the main factor explaining antibody retention, rather than current infection. In other words, antibody positivity may not be an effective marker of current toxoplasmosis and may instead be attributed to past exposure. However, the positive rate for an antibody may be an indirect indicator of the extent of toxoplasmosis infection in the environment or food.

Currently, Korea has no management standards for $T$. gondii in food. According to the Infectious Disease Web Statistics System (http://is.cdc.go.kr/nstat/index.jsp) of the KCDC, a few cases of $T$. gondii have been officially reported, despite no reports of foodborne toxoplasmosis. Considering the increasing meat consumption in Korea, it is necessary to monitor the occurrence of toxoplasmosis in retail meats. Therefore, a fast and reliable method for $T$. gondii detection in food can improve food safety in Korea.

$T$. gondii genotyping has been performed previously. Before 1997, the $B 1$ or $S A G 1$ genes were primarily used to detect toxoplasmosis; these loci are cannot be used to distinguish between types of T. gondii. In 1997, Howe and colleagues [15] developed a SAG2 genotype-based nested PCR assay; in an analysis of 68 infected patients, they were able to successfully distinguish between types of $T$. gondii, identifying 7 (10\%) type 1; 55 (81\%) type 2 , and $6(9 \%)$ type 3 strains. In addition, Ajzenberg et al. [16] evaluated 86 patients with congenital toxoplasmosis by MLST (multi-locus sequence typing) and found that 7 (8.1\%), $73(84.9 \%), 2(2.3 \%)$, and 4 patients $(4.7 \%)$ were positive for type $1,2,3$, and atypical strains, respectively.

In this study, we developed a method that combines conventional PCR with a PNA melting array to detect and genotype $T$. gondii. The method could distinguish between type 1,2, and 3 strains within a few hours and therefore has applications for real-time detection in food and clinical settings, since the toxicity of $T$. gondii varies among genotypes. In mouse studies, Khan et al. [17] and Sibley et al. [18] found that type 1 strains show the highest virulence and result in acute symptoms, while type 2 and 3 strains show low pathogenicity and result in chronic symptoms. In addition, Mordue et al. [19] reported that the strong toxicity of type 1 strains is caused by the Th1 immune response, leading to death by shock as the Th1 cytokine levels increases.

PNA is a DNA mimic, similar to LNA (locked nucleic 
Table 3. Melting temperature for the PNA probe and genotype calling.

\begin{tabular}{cccc}
\hline \multirow{2}{*}{ Match pattern } & \multicolumn{2}{c}{$\mathrm{T}_{\mathrm{m}}$ Range $\left({ }^{\circ} \mathrm{C}\right)$} & \multirow{2}{*}{ Call } \\
\cline { 2 - 3 } & GRA6-1 (FAM) & GRA6-2 (HEX) & \\
\hline Perfect match & $65-69$ & $65-69$ & 1 \\
Mismatch & $<64$ & $<64$ & 0 \\
\hline
\end{tabular}

acid) and MNA (morpholino nucleic acid). Its basic structure is composed of polyamides. As PNA has excellent affinity, selectivity, and high stability against nucleic acid-degrading enzymes, it freely decomposes by existing restriction enzymes [20]. Therefore, it is suitable for analytical studies and genetic detection. The PNA-DNA binding ability is superior to the DNA-DNA binding ability. A difference in melting temperature, i.e., about $10^{\circ} \mathrm{C}$ to $15^{\circ} \mathrm{C}$ in the case of a single nucleotide mismatch, can be clearly observed. SNPs, insertions, and deletions can be detected based on the difference in melting temperature. PNA has high thermal and chemical stability and are suitable for commercial kits, with a long shelf life. The hybridization efficiency is not dependent on the salt concentration. Therefore, false positive and false negative results are less likely to occur using PNA probes. Unlike other SNP detection probes, PNA is designed using relatively short sequences, such as 11- to 18-mers; accordingly, the probe design is simple [20]. The probe labeled with FAM at the 5 '-end (GRA6-1) was designed as a 14-mer, with a single nucleotide mismatch with the type 2 sequence, but an identical sequence to the sequences of type 1 and 3 strains. The GRA6-2 probe (5'HEX-labeled 16-mer) had a single mismatch in type 3 , but perfect matches to type 1 and 2 sequences.

In conclusion, this study provides proof-of-concept that the combination of conventional PCR and PNA melting array can be used for the fast, real-time detection and genotyping of $T$. gondii. Although the sensitivity of the conventional PCR requires improvement, the downstream PNA melting array was clearly able to accurately identify all three types of $T$. gondii with high selectivity. Future work will focus on increasing the detection sensitivity and broaden the applications of the assay.

\section{Acknowledgements}

This research was supported by the Korea Ministry of Food and Drug Safety (16161MFDS605).

\section{Conflict of Interest}

The authors have no financial conflicts of interest to declare.

\section{References}

1. Nielsen PE, Egholm M, Buchardt O. 1994. Peptide nucleic acid (PNA). A DNA mimic with a peptide backbone. Bioconjug. Chem. 5:3-7.

2. Egholm M, Buchardt O, Christensen L, Behrens C, Freier SM, Driver DA, et al. 1993. PNA hybridizes to complementary oligonucleotides obeying the Watson-Crick hydrogen-bonding rules. Nature 365: 566-568.

3. Stender H, Fiandaca M, Hyldig-Nielsen JJ, Coull J. 2002. PNA for rapid microbiology. J. Microbiol. Methods 48: 1-17.

4. Lehtola MJ, Torvinen E, Miettinen IT, Keevil CW. 2006. Fluorescence in situ hybridization using peptide nucleic acid probes for rapid detection of Mycobacterium avium subsp. avium and Mycobacterium avium subsp. paratuberculosis in potable-water biofilms. Appl. Environ. Microbiol. 72: 848-853.

5. Montoya JG, Liesenfeld O. 2004. Toxoplasmosis. Lancet (London, England). 363: 1965-1976.

6. Chai JY, Lin A, Shin EH, Oh MD, Han ET, Nam HW, et al. 2003. Laboratory passage and characterization of an isolate of Toxoplasma gondii from an ocular patient in Korea. Korean J. Parasitol. 41: 147-154.

7. Kim WJ, Yang S, Choi G, Moon BC, 2017. Peptide nucleic acid based molecular authentication for identification of four medicinal Paeonia species using melting array analysis of the internal transcribed spacer 2 region. Molecules 22: Pii E1922.

8. Gupta P, Roy J, Prasad M. 2001. Single nucleotide polymorphisms: a new paradigm for molecular marker technology and DNA polymorphism detection with emphasis on their use in plants. Curr. Sci. 80: 524-535.

9. Dubey J. 1998. Advances in the life cycle of Toxoplasma gondii. Int. J. Parasitol. 28: 1019-1024.

10. Song K-J, Shin J-C, Shin H-J, Nam H-W. 2005. Seroprevalence of toxoplasmosis in Korean pregnant women. Korean J. Parasitol. 43: 69-71.

11. Kim N-H, Kim H-R, Park H-S, Kim Y-S, Lee J-H. 2015. Seroprevalence of Coxiella burnetii and Toxoplasam gondii in cattle in Seoul. Korea. Korean J. Vet. Serv. 38: 233-239.

12. Cheong KS, An SC, Kim JO, Kim NS, Chang KH. 1994. A studies on toxoplasmosis antibody from slaughtered pigs and cattle. Korean J. Vet. Serv. 17: 32-36.

13. Jones JL, Dubey J. 2012. Foodborne toxoplasmosis. Clin. Infect. Dis. 55: 845-851.

14. Racka K, Bártová E, Budíková M, Vodrazka P. 2015. Survey of Toxoplasma gondii antibodies in meat juice of wild boar (Sus scrofa) in several districts of the Czech Republic. Ann. Agric. Environ. Med. 22: 231-235.

15. Howe DK, Honoré S, Derouin F, Sibley LD. 1997. Determination of genotypes of Toxoplasma gondii strains isolated from patients 
with toxoplasmosis. J. Clin. Microbiol. 35: 1411-1414.

16. Ajzenberg $D$, Cogné $N$, Paris $L$, Bessières $M H$, Thulliez $P$, Filisetti $D$, et al. 2002. Genotype of 86 Toxoplasma gondii isolates associated with human congenital toxoplasmosis, and correlation with clinical findings. J. Infect. Dis. 186: 684-689.

17. Khan A, Su C, German M, Storch G, Clifford D, Sibley LD. 2005. Genotyping of Toxoplasma gondii strains from immunocompromised patients reveals high prevalence of type I strains. J. Clin. Microbiol. 43: 5881-5887.
18. Sibley LD, Boothroyd JC. 1992. Virulent strains of Toxoplasma gondii comprise a single clonal lineage. Nature 359: 82-85.

19. Mordue DG, Monroy F, La Regina M, Dinarello CA, Sibley LD. 2001. Acute toxoplasmosis leads to lethal overproduction of Th1 cytokines. J. Immunol. 167: 4574-4584.

20. Komiyama M, Ye S, Liang X, Yamamoto $Y$, Tomita T, Zhou JM, et al. 2003. PNA for one-base differentiating protection of DNA from nuclease and its use for SNPs detection. J. Am. Chem. Soc. 125: 3758-3762. 